\title{
BMJ Open How have predictors, moderators, mediators, treatment response, remission and resistance been defined and measured in randomised controlled trials for adolescent depression? A scoping review protocol
}

Darren Courtney (D , , ${ }^{1,2}$ Priya Watson, ${ }^{1,2}$ Benjamin W C Chan, ${ }^{3}$ Kathryn J Bennett, ${ }^{4}$ Kirsten Neprily, ${ }^{5}$ Tabitha Zentner, ${ }^{1}$ Terri Rodak, ${ }^{6}$ Renira Narrandes, ${ }^{6}$ Peter Szatmari ${ }^{6}$

To cite: Courtney D, Watson P, Chan BWC, et al. How have predictors, moderators, mediators, treatment response, remission and resistance been defined and measured in randomised controlled trials for adolescent depression? A scoping review protocol. BMJ Open 2020;10:e36171. doi:10.1136/ bmjopen-2019-036171

- Prepublication history for this paper is available online. To view these files, please visit the journal online (http://dx.doi org/10.1136/bmjopen-2019036171).

Received 06 December 2019 Revised 19 May 2020 Accepted 20 May 2020
D) Check for updates

(c) Author(s) (or their employer(s)) 2020. Re-use permitted under CC BY-NC. No commercial re-use. See rights and permissions. Published by BMJ.

For numbered affiliations see end of article.

Correspondence to Dr Darren Courtney; dr.courtney.research@gmail. com

\section{ABSTRACT}

Introduction Among randomised controlled trials for depressed adolescents, the extent of variation in how depressive symptom outcomes are defined is unknown. The variability in which potential predictors of these outcomes are tested is also unclear. This paper is a protocol describing the methods of a planned scoping review. The scoping review will examine and summarise how change in depressive symptoms have been described in RCT treatment studies to date. This review will report the measures used to describe change in depressive symptoms and whether the measure was used as a continuous or binary outcome or both. This review will describe how dichotomous outcome terms are defined to describe change in depression severity. This review will also examine predictors, moderators and mediators of change in depressive symptoms within RCTs.

Methods and analysis In this paper, we describe the protocol for our scoping review. Following the format outlined by the Preferred Reporting Items for Systematic Reviews and Meta-Analyses extension for scoping reviews, a research librarian will develop an operationalised search strategy, which we will apply to the MEDLINE, Embase, PsycINFO and Cumulative Index to Nursing and Allied Health Literature databases. We will search for papers from inception to 6 February 2020. A hand search for key citations will also be conducted. Investigator-raters will screen articles, first via the titles and abstracts and then through full-text reviews. We will include articles with randomised control design which assess the treatment of adolescents with major depressive disorder. We will systematically extract and synthesise prespecified data which includes: definition of depression used for participant inclusion, measures used to evaluate changes in depression, type of outcome used (continuous, binary or both), definitions of dichotomous terms to denote change in depression (eg, response, remission, recovery, etc) and reported predictors/moderators/mediators of change. Ethics and dissemination Ethics approval is not required. Findings will be presented in journal publications and at conferences.

\section{Strengths and limitations of this study}

- This protocol follows the Preferred Reporting Items for Systematic Reviews and Meta-Analyses extension for scoping reviews checklist format to ensure best practice in conducting this review.

- We will use broad inclusion criteria and conduct our search using multiple databases, with publication dates starting at inception of the databases.

- We will use duplicate reviewers for data extraction.

- We will not be comprehensively examining grey literature or trial protocols.

- We will not be conducting a comprehensive risk of bias assessment on articles found or providing a synthesis of the evidence.

\section{INTRODUCTION}

Rationale

Depression is a common mental health disorder that often emerges in adolescence and can have serious and lifelong effects. The prevalence in adolescence is estimated at $11.7 \%^{1}$ in the USA and $7.6 \%^{2}$ in Canada. Depression ranks as the number one cause of disability adjusted life years and years lost to disability for adolescents, ${ }^{3}$ and depressed adolescents are at an increased risk of smoking, substance use and obesity, ${ }^{45}$ among other high-risk health behaviours. Depression is also a major risk factor for completed suicide. ${ }^{6}$ In the USA, suicide rates among adolescents have increased significantly in the past decade and is now the second leading cause of death in this age group. ${ }^{7}$ In order to prevent these complications and address the symptoms faced by depressed adolescents, optimal treatment is essential. 
Multiple randomised controlled trials (RCTs) have examined treatments for adolescent depression. ${ }^{89}$ Investigators have used a variety of approaches to describe change (or lack thereof), such as the use of continuous scales or of dichotomous terms like 'response', 'remission' and 'treatment resistance'. The extent of variability in the definitions of these outcome terms is unknown; neither is the rationale behind these definitions. There have been calls in the literature for greater standardisation of outcomes between clinical trials in order to optimise the use of the data collected and ongoing work is being done to set a standard for how outcomes in paediatric clinical trials should be reported. ${ }^{10-15}$ Proposed standards include: (1) specifying cut-offs and rationale for such cut-offs when continuous variables are being defined and analysed as categorical and (2) defining and justifying clinically meaningful change. ${ }^{16}$ Terminology that is consistent between studies, evidence-based and relevant for patients, families and clinicians is of prime importance in order to advance the field. In establishing a common set of terms and constructs, researchers can minimise inefficiencies in future studies and test the scientific validity of findings across multiple settings. Additionally, common terms would provide researchers, clinicians, patients and families with shared reference points when addressing decisions in care, enabling improved communication and clarity around treatment goals.

Guidelines for the treatment of adolescent depression have been well established with psychotherapy and antidepressant medications as foundational. ${ }^{17}$ Selective serotonin reuptake inhibitor medications and psychotherapy are first-line treatments. The Treatment for Adolescent Depression Study showed that a combination of these two modalities is most efficacious ${ }^{18}$; although, two other RCTs have challenged this notion. ${ }^{19}{ }^{20}$ Regardless, a significant minority of depressed adolescents do not meaningfully improve with first-line treatment interventions. ${ }^{21}$ Knowledge about who is likely to respond to which treatment and the mechanisms by which response occurs is a vital step in optimising treatment, with the eventual aim of increasing response rates and decreasing associated morbidity and mortality. These factors associated with response are termed 'predictors', 'moderators' or 'mediators' of response. Kraemer et al have operationally defined these terms: (1) a predictor is 'a baseline measure that has a main effect on outcome, but no interactive effect', (2) a moderator is 'a baseline or prerandomisation characteristic shown to have an interactive effect with treatment on the outcome' and (3) a mediator is 'an event or change occurring during treatment, (correlating) with treatment choice; hence, possibly be a result of treatment, and (has) either a main or interactive effect on the outcome'. ${ }^{22}$ A full assessment regarding the heterogeneity of the definition of 'response' across adolescent depression RCTs, therefore, also requires a full description of the predictors, moderators and mediators (PMMs) reported in these trials.
This paper outlines the protocol for a scoping review to address these issues. The scoping review has two main objectives:

Objective \#: to describe how change in depressive symptoms has been measured and reported in RCTs for the treatment of adolescent depression. Where dichotomous outcome terms are used (eg, 'response/non-response', 'remission', 'recovery', 'minimally important clinical difference' or 'treatment resistance'), to describe characteristics including: the measures used in the definition, cut-offs used in the degree of change required, the time period of reference and the rationale for the described definition.

Objective \#: to list the reported PMMs of change in depressive symptoms in the included RCTs. We will also comment on the extent to which these PMMs were hypothesised a priori and whether or not there were adjustments to the analyses to account for multiple testing-as each of these concepts are important potential contributors to bias in secondary analyses. These objectives lend themselves well to a scoping review as they aim to map concepts in literature. ${ }^{23}$

\section{METHODS}

\section{Protocol and registration}

This protocol follows the Preferred Reporting Items for Systematic Reviews and Meta-Analyses extension for scoping reviews (PRISMA-ScR) format. ${ }^{23}$ The guideline, developed by expert consensus, contains a checklist of 20 essential reporting items and 2 optional items, which should be part of all scoping review methodology. There is currently wide heterogeneity of scoping review methodologies, and these varying methodologies have been found to be of mixed quality. ${ }^{24}$ Adhering to the PRISMA-ScR guideline is intended to increase the methodological consistency and uptake of research findings across scoping reviews.

The protocol was registered in Open Science Framework on 24 April 2019 (https://osf.io/ubtcm).

\section{Study eligibility criteria}

Studies will be included if they meet the following criteria: (1) RCTs or secondary analyses of these trials. (2) Studies that assess the treatment of adolescents with depression. Treatment interventions may include pharmacological interventions (eg, medications, herbal supplements or other nutraceuticals), physiological interventions (eg, diet, exercise, sleep programmes, light therapy or acupuncture), psychosocial interventions (eg, psychotherapy, psychoeducation or other counselling), brain stimulation interventions (eg, repetitive transcranial magnetic stimulation, electroconvulsive therapy) or computerised interventions (eg, therapeutic online applications or videogames). We defined 'adolescence' as being between the ages of 13 and 17 years inclusive, and in order for the RCT to be included, $80 \%$ of participants will have to fall within that age range. If the 
article only reported a mean and $\mathrm{SD}$ of the age of the sample, an estimation of eligibility will be made based on the assumption of normal distribution of age within the sample. If the study had a broader age range, but still reported a subgroup analysis of adolescents with depression, the study will be included. We will include studies which define 'depression' according to diagnoses of major depressive disorder, dysthymia/persistent depressive disorder or depressive symptoms above an established cut-off on a measure. Studies where comorbidities we present with depression were included, so long as depression was the primary outcome as defined above. (3) Published in an English language journal. Exclusion criteria are: (1) protocol studies; (2) studies evaluating depression in the context of bipolar disorder, the peripartum period, premenstrual dysphoria, minor depression or seasonal affective disorder; (3) studies aimed at preventing depression or depression relapse; (4) studies focusing on cost-effectiveness.

\section{Information sources}

The search strategies will be drafted by an experienced librarian, and further refined by the research team. The librarian will combine subject heading and text terms related to depression, the adolescent age range and study type. The search strategy will be developed in MEDLINE and then modified appropriately for the remaining databases. The search strategy will be made available in the results manuscript. As per the research librarian's recommendations, we will identify potentially relevant documents through MEDLINE, Embase, PsycINFO and Cumulative Index to Nursing and Allied Health Literature from inception until 6 February 2020 to cover the time wherein much of the review was done. Citations from relevant articles found in our initial search will also be hand-searched. The final search results will be downloaded into the Covidence web application for storage and management, and duplicates will be removed.

\section{Selection of sources for evidence}

Two reviewers will be trained to screen articles based on title and abstract. At the screening stage, we will target high sensitivity for eligible articles, as a false negative classification would lead to a citation being excluded with no further review; whereas a false positive would move onto full-text screening and is less consequential. The lead investigators will rate and develop consensus on inclusion/exclusion of 100 citations, considered the 'reference standard'. The two other reviewers will then each independently rate the 100 citations from the reference standard list. We will aim for a sensitivity of the reviewers' ratings at least $80 \%$. Once this threshold is obtained by each reviewer, they will proceed with the initial screening.

Next, we will undertake full-text screening. Three reviewers will establish inter-rater reliability for full textscreening on 50 publications from the remaining list. We will aim for intraclass correlation coefficients of $>0.75$ as this is considered 'excellent' agreement. ${ }^{25}$ These three reviewers will then independently screen the articles based on the full text. We will identify disagreements on study selection and resolve differences by consensus and discussion with the third reviewer as necessary.

We will use a flow diagram to report and summarise numbers of sources of evidence screened, assessed for eligibility and included in the review, with reasons for exclusions at each stage (see figure 1).

\section{Data charting process}

A data extraction form will be developed by the two principal investigators in Microsoft Excel ${ }^{26}$ to track extracted variables from each study. Two of three reviewers will independently evaluate each of the eligible articles for data extraction, and data extraction forms will be compared. Any disagreements will be resolved through discussion between the two reviewers or further adjudication by the remaining third reviewer.

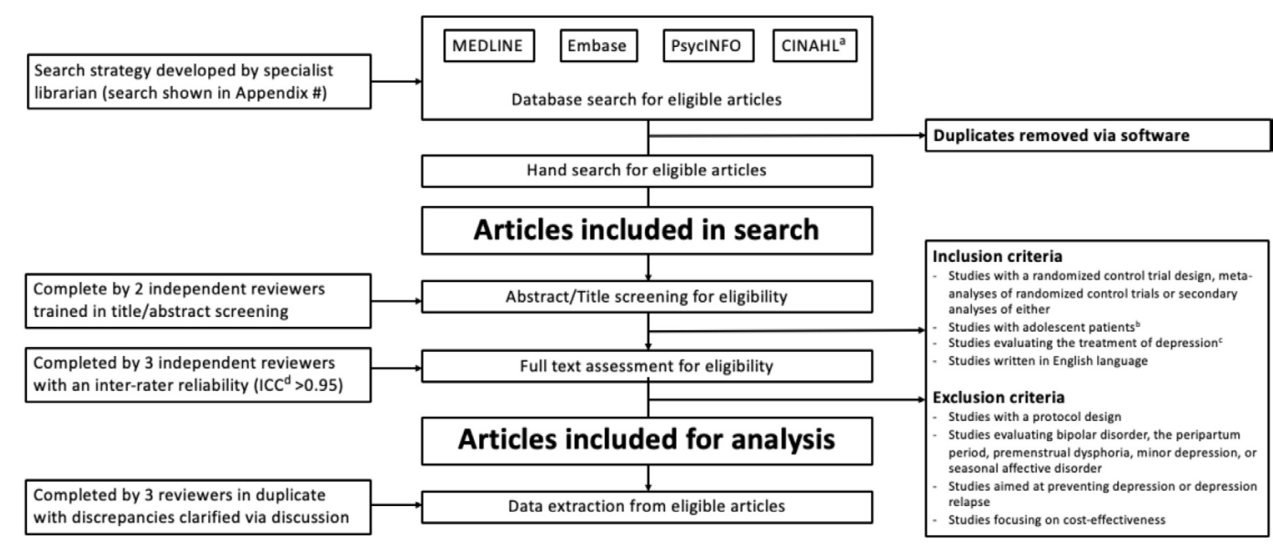

Figure 1 Preferred Reporting Items for Systematic Reviews and Meta-Analyses flow diagram for article selection. ${ }^{a} \mathrm{Cumulative}$ Index to Nursing and Allied Health Literature. ${ }^{b} 80 \%$ of subjects needed to fall within 13-17 years inclusive. ${ }^{\mathrm{C}}$ Inclusive of major depressive disorder, dysthymia/persistent depressive disorder or depressive symptoms above an established clinical cut-off on a measure. ${ }^{d}$ Intraclass correlation coefficient. 


\section{Data items and characteristic sources of evidence}

We will obtain data on the study (ie, country of origin, study design, funding source), cohort characteristics (sample size, study setting, female:male ratio) and treatment details (intervention employed, control comparator). We will report how investigators defined depression at baseline; that is, the definition for depression in their inclusion criteria and what measures were used. We will chart the measures used to describe change in depressive symptoms and whether the measure was used as a continuous or binary outcome or both. We will also extract terms used to describe dichotomous outcomes relating to changes in depressive symptoms, particularly scanning for the following terms: 'response/non-response', 'remission', 'recovery', 'minimal clinically important difference' and 'treatment resistance'. We will describe the definitions of these terms and the rationale for these definitions.

We will be extracting tested PMMs as per Kraemer et $a l$ 's definitions listed above. Secondary analyses will be grouped according to the primary study to which it refers. Where PMMs were found to be statistically significant, the effect size will be charted in whichever format it was described in the study (eg, ORs, Cohen's d, etc). For PMMs extracted, we will also chart whether they were reported as hypothesised prior to data collection, prior to initial data analysis, prior to secondary data analysis or during the secondary data analysis phase. Moreover, we will chart if the timing of the hypothesis was not reported at all. Furthermore, we will extract any reporting of efforts to adjust the analysis to account for bias from multiple testing (eg, Bonferroni corrections).

\section{Critical appraisal of individual sources of evidence}

As this is a scoping review intended to map and describe the extant literature, we will not undertake a detailed critical analysis of study quality and results.

\section{Synthesis of results}

For Objective 1, we will list the various terms used to describe change, how these terms were defined, which measures were used, how often the terms/definitions/ measures are used and which were chosen by larger studies (ie, $>100$ participants). Any narrative descriptions of the rationale for the use of these terms (or lack thereof) will also be included.

For Objective 2, we will list each of the PMMs tested for each original RCT how they were measured, whether or not they were found to be statistically significant, the size of the effect found, reporting of timing of hypotheses and any corrections for multiple testing.

\section{Patient and public involvement statement}

The current project is a scoping review of what is already in the literature. It does not involve the acquisition of new information. Patient and public involvement is not applicable in this situation.

\section{Ethics and dissemination}

This scoping review only involves the identification and synthesis of information in the reported literature. It does not involve extracting information at the individual participant level; as such, ethics approval is not required.

Once our review is completed, we intend to share this information with clinicians, health administrators and researchers through publications in high impact journals (eg, the Journal of the American Academy of Child and Adolescent Psychiatry) and conference presentations (eg, the American Academy of Child and Adolescent Psychiatry Annual Meeting, the Canadian Academy of Child and Adolescent Psychiatry Annual Meeting, the Society of Clinical Trials Annual Meeting and the Anxiety and Depression Association of America Annual Meeting). We will also develop a plain language summary, which will be posted on the Cundill Centre for Child and Youth Depression website. Both the summary and publications will be promoted through various communication channels (eg, Centre for Addiction and Mental Health Research Twitter account, with important stakeholders tagged; the Cundill Centre newsletter; emails to key stakeholders) using appropriate, tailored messaging. We will seek out relevant knowledge translation platforms to share our findings (eg, EENet Connect, an online community where members of Ontario's mental health and addictions system can post information; this platform allows members to pose questions and promotes discussion). The Cundill Centre knowledge broker will monitor conversations and evaluate the reach, usefulness and use of knowledge translation efforts (when possible) in alignment with the Cundill Centre's knowledge translation evaluation strategy.

We will also be using the findings from Objective 2 to incorporate the PMMs found in our scoping review in the data we collect for youth in our local longitudinal studies of depression. ${ }^{27}$ Furthermore, we will make recommendations for which PMMs to capture as data in longitudinal studies of depression. These recommendations will be made within our journal publications and conference presentations for use extended beyond our research centre.

\section{Limitations}

There will be some limitations to our methods. We will not be conducting a full risk of bias assessment. We will not be systematically reviewing protocols of RCTs, though we will look for these with larger published studies $(n>100)$. Some of the relevant information may not be listed in the main study paper, but rather in the protocols. We also did not include dissertations or other grey literature. We also only included English language articles. The above limitations are thought to be appropriate for our objectives and the intent to do a scoping review and not a systematic review with a focus on risk of bias.

Author affiliations

${ }^{1}$ Child, Youth and Family Services, Centre for Addiction and Mental Health, Toronto, Ontario, Canada 
${ }^{2}$ Psychiatry, University of Toronto, Toronto, Ontario, Canada

${ }^{3}$ Toronto, Ontario, Canada

${ }^{4}$ Health Research Methods, Evidence and Impact (formerly Clinical Epidemiology and Biostatistics), McMaster University Faculty of Health Sciences, Hamilton, Ontario,

Canada

${ }^{5}$ Psychology, University of Calgary, Calgary, Alberta, Canada

${ }^{6}$ Centre for Addiction and Mental Health, Toronto, Ontario, Canada

Acknowledgements We would like to thank Susan Dickens and Lisa Hawke for logistical support for this review. We would also like to thank Nancy Butcher for her input.

Contributors DC and PW first conceptualised the project, and will be acquiring, analysing and interpreting the data. BWCC, KN, TZ helped refine the conceptualisation of the project—particularly with regards to the methods section. They will also be involved in acquiring the data and interpreting the data. KJB and PS refined the conceptualisation of the project's objectives and overall design, as well as input on more specific methods. They will also assist with the interpretation of the findings. TR specifically assisted in developing the search strategy-which is a key aspect of the design of the study. RN helped conceptualise the knowledge translation strategy. DC, PW and BWCC drafted the manuscript. The remaining coauthors revised the manuscript critically for important intellectual content. All are in agreement to be accountable for all aspects of the work in ensuring that questions related to the accuracy or integrity of any part of the work are appropriately investigated and resolved. All will be involved in final approval of the published version

Funding This study was funded by Cundill Centre for Child and Youth Depression. Competing interests None declared.

Patient and public involvement Patients and/or the public were not involved in the design, or conduct, or reporting, or dissemination plans of this research.

Patient consent for publication Not required.

Provenance and peer review Not commissioned; externally peer reviewed.

Open access This is an open access article distributed in accordance with the Creative Commons Attribution Non Commercial (CC BY-NC 4.0) license, which permits others to distribute, remix, adapt, build upon this work non-commercially, and license their derivative works on different terms, provided the original work is properly cited, appropriate credit is given, any changes made indicated, and the use is non-commercial. See: http://creativecommons.org/licenses/by-nc/4.0/.

ORCID iD

Darren Courtney http://orcid.org/0000-0003-1491-0972

\section{REFERENCES}

1 Mojtabai R, Olfson M, Han B. National trends in the prevalence and treatment of depression in adolescents and young adults. Pediatrics 2016;138:peds.2016-1878.

2 Cheung $\mathrm{AH}$, Dewa CS. Canadian community health survey: major depressive disorder and suicidality in adolescents. Healthc Policy 2006;2:76-89.

3 Gore FM, Bloem PJN, Patton GC, et al. Global burden of disease in young people aged 10-24 years: a systematic analysis. Lancet 2011;377:2093-102.

4 Johnson D, Dupuis G, Piche J, et al. Adult mental health outcomes of adolescent depression: a systematic review. Depress Anxiety 2018;35:700-16.

5 Isa A, Bernstein I, Trivedi M, et al. Understanding the impact of treatment on the dimensions of childhood depression. J Child Adolesc Psychopharmacol 2017;27:160-6.

6 Renaud J, Berlim MT, McGirr A, et al. Current psychiatric morbidity, aggression/impulsivity, and personality dimensions in child and adolescent suicide: a case-control study. J Affect Disord 2008;105:221-8.

7 Curtin SC, Heron MP. Death rates due to suicide and homicide among persons aged 10-24: United States, 2000-2017. CDC report, 2019. Available: https://www.cdc.gov/nchs/data/databriefs/db352-h. pdf [Accessed 29 Nov 2019].

8 Cipriani A, Zhou X, Del Giovane C, et al. Comparative efficacy and tolerability of antidepressants for major depressive disorder in children and adolescents: a network meta-analysis. Lancet 2016;388:881-90.

9 Weersing VR, Jeffreys M, MT D, et al. Evidence base update of psychosocial treatments for child and adolescent depression evidence base update of psychosocial treatments for child and adolescent depression. J Clin Child Adolesc Psychol 2016;00:1-33.

10 Sinha I, Jones L, Smyth RL, et al. A systematic review of studies that aim to determine which outcomes to measure in clinical trials in children. PLoS Med 2008;5:e96-578.

11 Szatmari P, Offringa M, Butcher NJ, et al. Counting what counts: the case for harmonized outcomes in child and youth mental health research. J Am Acad Child Adolesc Psychiatry 2019;58:656-8.

12 Idzerda L, Rader T, Tugwell P, et al. Can we decide which outcomes should be measured in every clinical trial? A scoping review of the existing conceptual frameworks and processes to develop core outcome sets. J Rheumatol 2014;41:986-93.

13 Williamson $\mathrm{P}$, Altman D, Blazeby J, et al. Driving up the quality and relevance of research through the use of agreed core outcomes. $J$ Health Serv Res Policy 2012;17:1-2.

14 Gorst SL, Gargon E, Clarke M, et al. Choosing important health outcomes for comparative effectiveness research: an updated review and identification of gaps. PLoS One 2016;11:e0168403.

15 Krause KR, Bear HA, Edbrooke-Childs J, et al. Review: what outcomes count? A review of outcomes measured for adolescent depression between 2007 and 2017. J Am Acad Child Adolesc Psychiatry 2019;58:61-71.

16 Kapadia M, Thurairajah P, Offringa M. Draft InsPECT candidate reporting items., 2019. Available: https://osf.io/zyr9k/ [Accessed 01 Dec 2019].

17 NICE. Depression in children and young people: identification and management.NICE guideline [NG134. National Institute for Health and Care Excellence, 2019.

18 March J, Silva S, Petrycki S, et al. Fluoxetine, cognitive-behavioral therapy, and their combination for adolescents with depression: treatment for adolescents with depression study (TADS) randomized controlled trial. JAMA 2004;292:807-20.

19 Goodyer IM, Dubicka B, Wilkinson $\mathrm{P}$, et al. A randomised controlled trial of cognitive behaviour therapy in adolescents with major depression treated by selective serotonin reuptake inhibitors. The adapt trial. Health Technol Assess 2008;12:hta12140.

20 Davey CG, Chanen AM, Hetrick SE, et al. The addition of fluoxetine to cognitive behavioural therapy for youth depression (YoDA-C): a randomised, double-blind, placebo-controlled, multicentre clinical trial. Lancet Psychiatry 2019;6:735-44.

21 Maalouf FT, Atwi M, Brent DA. Treatment-Resistant depression in adolescents: review and updates on clinical management. Depress Anxiety 2011;28:946-54.

22 Kraemer HC, Wilson GT, Fairburn CG, et al. Mediators and moderators of treatment effects in randomized clinical trials. Arch Gen Psychiatry 2002;59:877-83.

23 Tricco AC, Lillie E, Zarin W, et al. PRISMA extension for scoping reviews (PRISMA-ScR): checklist and explanation. Ann Intern Med 2018;169:467-73.

24 Tricco AC, Lillie E, Zarin W, et al. A scoping review on the conduct and reporting of scoping reviews. BMC Med Res Methodol 2016;16:15.

25 Fleiss JL, Levin B, Paik MC. Statistical Methods for Rates and Proportions. John Wiley \& Sons, 2013.

26 Microsoft excel. version 14.7.7 Microsoft Corporation. 2011.

27 Courtney DB, Cheung A, Henderson J, et al. Effectiveness of an integrated care pathway for adolescents with depression: a pilot clinical trial protocol. J Can Acad Child Adolesc Psychiatry 2019;28:115-33. 